\title{
The important role of general practice in the care of cancer survivors
}

Michael Jefford, Bogda Koczwara, Jon Emery, Elysia Thornton-Benko, Janette Vardy

\section{Background}

The number of people living with and beyond cancer is increasing substantially. Primary care has an important role in the ongoing management of cancer survivors.

\section{Objective}

The aim of this article is to outline common concerns of cancer survivors, evidence to support the role of general practitioners (GP) in survivorship care and key aspects of primary care-led survivorship care.

\section{Discussion}

Clinical trials have shown that, in particular circumstances and with well-designed models, GP-led care is as effective as oncology specialist-led care. Regardless of the model of care, general practice has key roles in care coordination, management of multimorbidity, secondary prevention and health promotion, management of psychosocial care and promotion of self-management. Communication and collaboration between GPs and specialist cancer services is critical to support patients and healthcare providers in the delivery of care.
IN AUSTRALIA, the number of people living with or beyond cancer (defined as 'cancer survivors') is increasing because of population ageing, increased cancer detection and improved treatments. In 2014 there were estimated to be almost 1.1 million Australians (approximately 1 in 22) with a personal history of cancer. ${ }^{1}$ This number is projected to increase to 1.9 million by 2040 . The majority are long-term (>5 years) survivors. ${ }^{1}$ The most prevalent groups are those with a personal history of prostate, breast or colorectal cancer or melanoma. ${ }^{1}$ Most are $>70$ years of age and have other comorbid conditions. ${ }^{2}$

Survivors may experience a range of consequences of cancer and cancer treatments. ${ }^{2-5}$ Many of these issues are common across cancer types and include: persistent physical symptoms, such as fatigue, pain, insomnia and difficulties with memory and thinking; psychological issues, such as anxiety and fear of cancer recurrence; and practical consequences, such as difficulty resuming work and altered relationships. ${ }^{2-5}$ Many of these issues may be present for years after completing treatments. ${ }^{2,4}$ Symptom burden is greater in survivors when compared with the general population, and survivors report inferior quality of life. ${ }^{2-5}$ Survivors may have other health risk factors, such as being inactive or overweight, or smoking. ${ }^{5}$ Survivors report a lack of information about the post-treatment phase and strategies to remain well; a predominant unmet need relates to the management of psychological worries. ${ }^{3,4,6}$ Some survivors are at risk of developing 'late effects' that may develop years after cancer treatment, including organ damage (eg cardiac failure) or another primary cancer.

Survivors may not be aware of their health risks and may feel unprepared for the post-treatment phase. ${ }^{3,7}$ General practitioners (GPs) have an important role in the care of these patients. ${ }^{8,9}$ In addition to a role in cancer surveillance, GPs have key roles in the management of comorbid illness, secondary prevention, health promotion, self-management promotion and coordination of care, which are usual roles for GPs.

\section{Existing and recommended models of care}

Current models of post-treatment care tend to be oncology-led and focus on surveillance for cancer recurrence. Unfortunately, these models leave survivors with significant unmet needs. They also fail to address many aspects of holistic care. These models are not sustainable given the rising numbers of survivors and limited health workforce, and do not represent the best use of oncology specialist expertise. ${ }^{7}$

To overcome these limitations, there have been a number of consensus 
recommendations outlining best principles for survivorship care.

The influential US Institute of Medicine report from 2006 suggested that follow-up should serve four broad goals: 1) prevention of recurrent and new cancers, and of the late effects of cancer treatments; 2) surveillance, not just for cancer recurrence or other cancers, but also for possible late effects; 3) interventions to deal with consequences, such as symptoms, distress and concerns related to practical issues such as return to work; and 4) coordination between all providers to ensure that survivors' needs are met. ${ }^{2}$

Cancer Australia has developed Principles of Cancer Survivorship, ${ }^{10}$ and the Clinical Oncology Society of Australia (COSA) has a recommended Model of Cancer Survivorship Care. ${ }^{11,12}$ The COSA model recognises that the most appropriate model of care will be determined by factors such as the type of cancer and treatments, current symptoms and concerns, the risk of later problems, time since completing treatments, practical issues such as the relationship with primary care providers, and patient and provider preferences. Survivors should be supported to self-manage, and be linked to other community-based providers. Cancer Australia recommends that breast cancer survivors are offered shared post-treatment care between oncology and primary care providers; ${ }^{13}$ this model could be applicable to other cancers. Australian Optimal Care Pathways (OCPs) have been developed for many cancer types and are consistent with these guidelines. The OCPs describe the key steps in a patient's cancer journey and expected standards of care. They were developed through consultation with a wide range of expert multidisciplinary teams, peak health organisations, consumers and carers, and are endorsed by the National Cancer Expert Reference Group, Cancer Australia and Cancer Council Australia.

Several trials have shown that cancer survivors can be safely and effectively managed by GPs. ${ }^{8}$ These trials have tested models of either shared care or direct transfer of follow-up to the
GP. Systematic reviews have found no differences between hospital follow-up and alternative models involving GPs for a range of outcomes including patient quality of life, satisfaction with care and clinical outcomes including detection of recurrence. ${ }^{14,15}$ These trials were not powered to examine clinical outcomes such as survival or time to detection of recurrence. The core components of an effective model of follow-up involving GPs include: role clarity, clear guidance for GPs on management of common problems, effective two-way communication and accessible specialist care when required. ${ }^{8}$

Breast cancer follow-up has been the most extensively studied; in Australia, several pilot programs have applied evidence from international trials to develop a national model of shared care. ${ }^{13}$ An Australian trial of GP-led follow-up for colorectal cancer showed differences in the types of surveillance tests ordered but no differences in psychosocial or clinical outcomes. ${ }^{16}$ GP-led follow-up for melanoma survivors also appears feasible and safe. ${ }^{17}$

The only trial of shared care for prostate cancer, ProCare, found no differences in psychosocial outcomes or clinical care, though shared care was less expensive and preferred by men. ${ }^{18}$ A larger trial of shared care for colorectal cancer is in progress in the state of Victoria. ${ }^{19}$ Shared care is increasingly being implemented, particularly for survivors of breast, bowel and prostate cancer. As the number of cancer survivors increases, it is likely that these models of cancer care will be more widely implemented and expanded to other cancers. It will be important to examine and share findings from the routine implementation of GP-led and shared care models.

\section{Integrating survivorship care with management of comorbid illness}

Cancer frequently coincides with other long-term diseases, reflecting the high prevalence of chronic conditions in Australia and common risk factors for cancer and other chronic conditions, including age.$^{20}$ Many cancer treatments and lifestyle factors, such as poor diet and inactivity associated with cancer treatment, increase the severity of pre-existing comorbidities or risk of developing new chronic conditions. ${ }^{21}$

GPs play a critical part in ensuring that survivorship care is integrated into the overall care of patients with cancer, contributing their expertise in chronic disease management. Excellent communication between the GP and oncology teams is paramount to achieve optimal cancer treatment and outcomes, and holistic evidence-based care.

During cancer treatment, patients and clinicians may decrease their attention on managing other chronic diseases. Survivors have a higher risk of mental health problems and cardiovascular, musculoskeletal and endocrine conditions, and the presence of comorbidity correlates with the perception of poor health. ${ }^{22}$

Comorbidity is particularly common in Aboriginal and Torres Strait Islander peoples and patients with lower socioeconomic status, for whom cancer outcomes are already inferior. This underscores the importance of directing interventions to these population subgroups to ensure comorbidities are managed in an integrated way prior to, during and after cancer treatments. ${ }^{23}$

Specific tools are needed to assist healthcare providers to manage comorbidities and cancer, including risk calculators that quantify the risk of specific comorbidities after certain cancer treatments and tools to guide optimal management. Behavioural and lifestyle approaches and self-management strategies are essential elements of survivorship care..$^{2,11,12}$ GPs are well placed to provide such comprehensive management oversight, with ongoing communication between the GP and oncologist, incorporating other non-GP specialists as indicated.

\section{Health promotion and lifestyle modification}

The GP is ideally placed to assist survivors to modify their lifestyles 
and reduce the risk of a new primary cancer or cancer recurrence. GPs can also assist with decreasing treatment side-effects and long-term sequelae associated with cancer and cancer treatment, such as metabolic syndrome, cardiovascular disease, osteoporosis and psychosocial issues.

The detrimental effects of smoking and excessive alcohol intake - and their associations with cancer - are well accepted, and smoking cessation in particular is frequently addressed by GPs. Additional benefits of smoking cessation for cancer survivors include decreased mortality, ${ }^{24}$ reduced risk of a second malignancy and improved quality of life. ${ }^{25}$ Despite this, a large US epidemiological study found that only $51 \%$ of cancer patients who smoked reported being counselled to cease within the previous year. ${ }^{26}$ There is an increased risk of a new primary cancer and poorer mortality in people who drink alcohol and have cancers such as head and neck cancer. ${ }^{27}$ For those with moderate alcohol intake and more common cancers such as breast and colorectal cancer, some studies suggest that cancer-specific mortality may be higher for patients who consume alcohol. ${ }^{27}$ Despite this, an Australian study found that many GPs did not routinely discuss alcohol consumption with patients. ${ }^{28}$

Large epidemiological studies have shown that physical activity, particularly a combination of aerobic and resistance exercise, can decrease the risk of several cancers and the risk of cancer recurrence. ${ }^{29,30}$ The COSA Exercise Position Statement recommends that exercise be embedded into cancer care, with best practice to include referral of the patient to an exercise specialist with cancer experience. ${ }^{31}$ GPs are encouraged to 'discuss, recommend and refer'. ${ }^{31}$ It is recognised that, currently, there is an insufficient number of exercise specialists with cancer experience.

Obesity is a risk factor for some common cancers (eg breast, colon) and is associated with poorer survival and a higher risk of cancer recurrence than for people of normal weight. ${ }^{32}$ The GP can facilitate lifestyle modification through enhanced care plans, with referral of survivors to allied health professionals such as exercise specialists, dietitians and clinical psychologists; referral to community-based programs; and promotion of the benefits of exercise, healthy eating and weight management.

\section{Conclusion}

With the growing number of cancer survivors, and evidence that oncologistled care can be suboptimal, new models of care should be implemented. GP-led follow-up and shared care models offer promise of more holistic, coordinated and accessible care and may be appropriate for many survivors. GPs have important roles in the management of comorbid illness, symptom management, psychosocial care, secondary prevention, health promotion and overall care coordination. Communication and collaboration between GPs and other primary care providers and specialist cancer services is critical to support patients and healthcare providers in delivery of care.

Numerous resources exist to support survivorship care; please refer to the 'Resources' section.

\section{Key points}

- There is a large and growing number of cancer survivors in Australia.

- Survivors may experience a broad range of consequences from cancer and cancer treatments.

- Cancer survivorship care is increasingly seen as akin to other chronic disease management.

- Primary care has a critical role in care coordination, management of multimorbidity and secondary prevention.

- Primary care offers the opportunity for greater access to care.

- Trials have shown that GP-led follow-up and models of shared care are as effective as oncology specialist-led care for particular patient groups.

- Primary care providers should have a greater role in the management of survivors.

\section{Resources}

\section{Information for patients, survivors and carers}

- Cancer Council: High-quality, evidencebased resources in many languages, www.cancer.org.au/about-cancer/ after-treatment

\section{Online cancer survivorship education for primary care professionals}

- Future Learn-Cancer Survivorship for Primary Care Practitioners, www. futurelearn.com/courses/cancersurvivorship

- eviQ Education: Cancer survivorship introductory course, https://education. eviq.org.au/courses/supportive-care/ cancer-survivorship-introductory-course

\section{Australian cancer guidelines}

- Cancer Council - Primary care resources, https://cancer.org.au/ health-professionals/primary-careresources

- Cancer Council Australia - Cancer Council Australia's Clinical Guidelines Network platform, https://wiki.cancer. org.au/australia/Guidelines

- Cancer Council - Optimal cancer care pathways, www.cancer.org.au/ health-professionals/optimal-cancercare-pathways.html

- Clinical Oncology Society of AustraliaCOSA Model of Survivorship Care, www. cosa.org.au/groups/survivorship/resources

- Cancer Australia - Principles of cancer survivorship, https://canceraustralia. gov.au/publications-and-resources/ cancer-australia-publications/ principles-cancer-survivorship

\section{Other Australian resources}

- Peter MacCallum Cancer Centre Australian Cancer Survivorship Centre: Resources for health professionals, survivors and carers, www.petermac.org/ cancersurvivorship

- Peter MacCallum Cancer Centre MyCarePlan.org.au: Survivorship care plan generator for people treated for early stage breast, bowel or prostate cancer, www.mycareplan.org.au

- Faculty of Radiation Oncology, The Royal Australian and New Zealand 


\section{College of Radiologists: Information for general practitioners, including about side effects and follow-up care, www.targetingcancer.com.au}

\section{Authors}

Michael Jefford MBBS, MPH, MHIthServMt, PhD, FRACP, Consultant Medical Oncologist, Department of Medical Oncology, Peter MacCallum Cancer Centre, Vic; Director, Australian Cancer Survivorship Centre, A Richard Pratt Legacy, Peter MacCallum Cancer Centre, Vic; Lead, Survivorship and Living Well After Cancer Research, Department of Cancer Experiences Research, Peter MacCallum Cancer Centre, Vic; Professor, Sir Peter MacCallum Department of Oncology, University of Melbourne. Michael.Jefford@petermac.org

Bogda Koczwara BMBS, MBioethics, FRACP, Professor of Cancer Medicine, Flinders University, SA; Senior Staff Specialist, Department of Medical Oncology, Flinders Medical Centre, SA Jon Emery MA, MBBCh, MRCGP, FRACGP, DPhil, Herman Professor of Primary Care Cancer Research, Department of General Practice, and Centre for Cancer Research, University of Melbourne, Vic Elysia Thornton-Benko BSc, MBBS (Hons), PhD, FRACGP, Specialist General Practitioner, NSW Janette Vardy BMed (Hons), FRACP, PhD, Professor of Cancer Medicine, Faculty of Medicine and Health, University of Sydney, NSW; Concord Cancer Centre, Concord Repatriation General Hospital, NSW Competing interests: BK is supported by a National Breast Cancer Foundation Practitioner Fellowship. $\mathrm{JE}$ is an National Health and Medical Research Council (NHMRC) Practitioner Fellow.

Funding: None.

Provenance and peer review: Not commissioned, externally peer reviewed.

\section{References}

1. Cancer Council Australia. Australians living with and beyond cancer in 2040. Sydney: Cancer Council Australia, 2018. Available at www.cancer. org.au/content/pdf/News/MediaReleases/2018/ Prevalence\%20in\%202040 FINAL.PDF [Accessed 31 March 2020].

2. Institute of Medicine and National Research Council. From cancer patient to cancer survivor: Lost in transition. Washington, DC: The National Academies Press, 2006.

3. Jefford M, Karahalios E, Pollard A, et al. Survivorship issues following treatment completion - Results from focus groups with Australian cancer survivors and health professionals. J Cancer Surviv 2008;2(1):20-32. doi: 10.1007/s11764-008-0043-4.

4. Jefford M, Ward AC, Lisy K, et al. Patient-reported outcomes in cancer survivors: A population-wide cross-sectional study. Support Care Cancer 2017;25(10):3171-79. doi: 10.1007/s00520-0173725-5.

5. Tan SY, Turner J, Kerin-Ayres K, et al. Health concerns of cancer survivors after primary anti-cancer treatment. Support Care Cancer 2019;27(10):3739-47. doi: 10.1007/s00520-01904664-w.

6. Lisy K, Langdon L, Piper A, Jefford M. Identifying the most prevalent unmet needs of cancer survivors in Australia: A systematic review. Asia Pac J Clin Oncol 2019;15(5):e68-e78. doi: 10.1111/ ajco.13176.
7. Alfano CM, Jefford M, Maher J, Birken SA, Mayer DK. Building personalized cancer follow-up care pathways in the United States: Lessons learned from implementation in England, Northern Ireland, and Australia. Am Soc Clin Oncol Educ Book 2019;39:625-39. doi: 10.1200/ EDBK_238267.

8. Emery JD, Shaw K, Williams B, et al. The role of primary care in early detection and follow-up of cancer. Nat Rev Clin Oncol 2014;11(1):38-48. doi: 10.1038/nrclinonc.2013.212.

9. Rubin G, Berendsen A, Crawford SM, et al. The expanding role of primary care in cancer control. Lancet Oncol 2015;16(12):1231-72. doi: 10.1016/ S1470-2045(15)00205-3.

10. Cancer Australia. Principles of Cancer Survivorship. Surry Hills, NSW: Cancer Australia, 2017.

11. Clinical Oncology Society of Australia Model of Survivorship Care Working Group. Model of survivorship Care: Critical components of cancer survivorship care in Australia position statement. Sydney: Clinical Oncology Society of Australia, 2016.

12. Vardy JL, Chan RJ, Koczwara B, et al. Clinical Oncology Society of Australia position statement on cancer survivorship care. Aust J Gen Pract 2018;48(12):833-36. doi: 10.31128/ AJGP-07-19-4999.

13. Cancer Australia. Cancer Australia statement Influencing best practice in breast cancer. Surry Hills, NSW: Cancer Australia, 2016.

14. Lewis RA, Neal RD, Williams $\mathrm{NH}$, et al. Follow-up of cancer in primary care versus secondary care: Systematic review. Br J Gen Pract 2009;59(564):e234-47. doi: 10.3399/ bjgp09X453567.

15. Zhao Y, Brettle A, Qiu L. The effectiveness of shared care in cancer survivors - A systematic review. Int J Integr Care 2018;18(4):2. doi: 10.5334/ ijic.3954.

16. Wattchow DA, Weller DP, Esterman A, et al. General practice vs surgical-based follow-up for patients with colon cancer: Randomised controlled trial. Br J Cancer 2006;94(8):1116-21. doi: 10.1038/ sj.bjc. 6603052

17. Murchie P, Nicolson MC, Hannaford PC, Raja EA, Lee AJ, Campbell NC. Patient satisfaction with GP-led melanoma follow-up: A randomised controlled trial. Br J Cancer 2010;102(10):1447-55. doi: 10.1038/sj.bjc.6605638.

18. Emery JD, Jefford M, King M, et al. ProCare trial: A phase II randomized controlled trial of shared care for follow-up of men with prostate cancer. BJU Int 2017;119(3):381-89. doi: 10.1111/bju.13593.

19. Jefford M, Emery J, Grunfeld E, et al. SCORE: Shared care of colorectal cancer survivors: Protocol for a randomised controlled trial. Trials 2017;18:506. doi: 10.1186/s13063-017-2245-4.

20. Edwards BK, Noone AM, Mariotto AB, et al. Annual Report to the Nation on the status of cancer, 1975-2010, featuring prevalence of comorbidity and impact on survival among persons with lung, colorectal, breast, or prostate cancer. Cancer 2014;120(9):1290-314. doi: 10.1002/ cncr.28509.

21. Yabroff KR, Lawrence WF, Clauser S, Davis WW, Brown ML. Burden of illness in cancer survivors: Findings from a population-based national sample. J Natl Cancer Inst 2004;96(17):1322-30. doi: 10.1093/jnci/djh255.

22. Ng HS, Koczwara B, Roder D, Vitry A. Changes in the prevalence of comorbidity in the Australian population with cancer, 2007-2014. Cancer Epidemiol 2018;54:56-62. doi: 10.1016/j. canep.2018.03.010.
23. Louwman WJ, Aarts MJ, Houterman S, van Lenthe FJ, Coebergh JW, Janssen-Heijnen ML. A 50\% higher prevalence of life-shortening chronic conditions among cancer patients with low socioeconomic status. Br J Cancer 2010;103(11):1742-48. doi: 10.1038/sj.bjc.6605949.

24. Browman GP, Wong G, Hodson I, et al. Influence of cigarette smoking on the efficacy of radiation therapy in head and neck cancer. N Engl J Med 1993;328(3):159-63. doi: 10.1056/ NEJM199301213280302.

25. Gritz ER, Carmack CL, de Moor C, et al. First year after head and neck cancer: Quality of life. J Clin Oncol 1999;17(1):352-60. doi: 10.1200/ JCO.1999.17.1.352.

26. Ramaswamy AT, Toll BA, Chagpar AB, Judson BL. Smoking, cessation, and cessation counseling in patients with cancer: A population-based analysis. Cancer 2016;122(8):1247-253. doi: 10.1002/ cncr.29851.

27. LoConte NK, Brewster AM, Kaur JS, Merrill JK, Alberg AJ. Alcohol and cancer: A Statement of the American Society of Clinical Oncology. J Clin Oncol 2018;36(1):83-93. doi: 10.1200/ JCO.2017.76.1155.

28. Wellard L, Corsini N, Hughes C. Discussing alcohol and cancer with patients: Knowledge and practices of general practitioners in New South Wales and South Australia. Aust Fam Physician 2016;45(8):588-993.

29. World Cancer Research Fund/American Institute for Cancer Research. Continuous Update Project Expert Report. Physical activity and the risk of cancer. London: World Cancer Research Fund International, 2018. Available at www.wcrf.org/ dietandcancer [Accessed 10 February 2020].

30. Schmid D, Leitzmann MF. Association between physical activity and mortality among breast cancer and colorectal cancer survivors: A systematic review and meta-analysis. Ann Oncol 2014;25(7):1293-311. doi: 10.1093/annonc/mdu012.

31. Cormie P, Atkinson M, Bucci L, et al. Clinical Oncology Society of Australia position statement on exercise in cancer care. Med J Aust 2018;209(4):184-87. doi: 10.5694/mja18.00199.

32. Ligibel JA, Alfano CM, Courneya KS, et al. American Society of Clinical Oncology position statement on obesity and cancer. J Clin Oncol 2014;32(31):3568-74. doi: 10.1200/ JCO.2014.58.4680. 